\title{
Image Fusion on Coloured and Gray Scale Multi Focus Images by using Hybrid DWT-DCT
}

\author{
Mamta Sharma \\ M.Tech Student (C.S.) \\ GITS Udaipur, India
}

\author{
Sarika Khandelwal \\ Associate Professor \\ GITS Udaipur, India
}

\begin{abstract}
Any piece of information is meaningful only when it is able to convey the content about it. The clarity and quality of information is important. Image Fusion is a technique to improve the quality and quantity of information from a set of images. By the process of image fusion the more information from each of the given images is combining together to generate a resultant image whose quality is maximum to any of the input images.

We proposed a Hybrid DWT-DCT method to fuse multi focus images. In this technique we convert the image data from spatial domain to transform domain.. Then decompose the transform data into four parts that is LL, LH, HL, HH part. This decomposition Process again applies in LL part at two levels. After this three level decomposition we combine the input image data by applying average method using DCT and get fused data. This data are in transform domain again convert to spatial domain by applying IDCT and IDWT method and get final fused image with better visual Quality. After getting the result of fused image we compare the quality measure parameters of different technique like PCA, DCT, average pixel, maximum pixel, minimum pixel, HDWT method to hybrid DWT-DCT method. And conclude that the PSNR value and Entropy of fused image have better result as compare to other techniques. Due to this the fused image has better visual Quality as well as more informative data would contain in fused image.
\end{abstract}

\section{Keywords}

Multi focus image, Fused Image, PSNR, Entropy, DWT, DCT, PCA, HDWT, Hybrid DWT-DCT

\section{INTRODUCTION}

In applications of digital cameras, when a lens focuses on a object at a given distance, all objects at same distance are sharply focused. Objects are not at the same distance out of focus and given objects are not sharp. It is generally not possible to capture an image that contains all relevant objects in focus. One way to overcome this problem is combining multiple image of same scene that is image fusion; in which one can capture a series of pictures with different focus settings and combine them to produce an image with sharply focus on each object. During fusion process, all the important visual information occurs in the input images must be transferred into fused image without introduction of distortion. In this field the fusion algorithm should be reliable and robust to distortion such as noise or mis-registration. Image fusion is a branch of data fusion where data occurs in the form of arrays of numbers that represent brightness, distance, color, temperature, and other important properties. Such data can be two-dimensional (multi focus images), three-dimensional (audio, video sequences or volumetric images), or higher dimensions. Last decades, multi variance imaging techniques have become an important source of information to aid diagnosis in many medical fields

\section{PROCESS OF IMAGE FUSION}

Image fusion process is implemented to obtain a better visual quality of certain phenomena, and to introduce and enhance intelligence or system control functions. These processes apply on two multi focal images of same scene and combine them by using fusion algorithm. After completing all the steps of fusion algorithm we get a fused image. These processes are helpful for celerity of multi focus image of same scene and make a visibility more for blurred images. In this process we are taking two images. This is blurred by distortion of image. Due to this images are not clearly visible. For that we combine this type of images by using different algorithms like PCA, DWT, DCT, Averaging method.

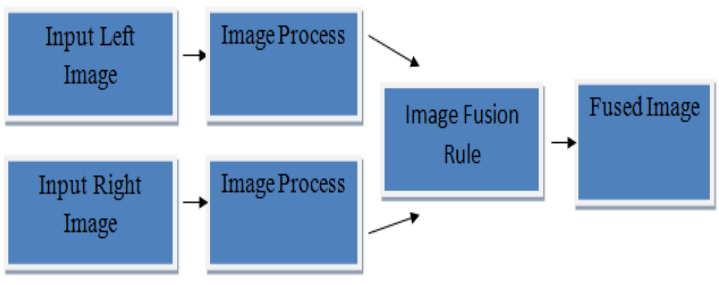

Figure 1 Flow Diagram of Image Fusion Process

The Above method is easiest process of image fusion. This is the block diagram of image fusion. In which we are collecting two multifocal images and process them by using PCA, DCT, DWT and apply for fusion rule like averaging, maximum and minimum method.

After the process of fusion rule we get a fused image. This result image has a better quality as compare to input image. The process of merging of two images or more than two images to get better quality image or it is a process of combining the complementary as well as the common features of set of similar incomplete or distorted images, to provide a resultant image. The requirement of image fusion mechanism for high resolution on multispectral images, panchromatic and real world images for better vision of image.

\section{TYPES OF IMAGE FUSION}

The set of images of same scene are fused together to generate a new single image with optimum information content. For example in noise full environment and illumination variant a human operators like detector tool may not be able to detect objects of his interest after that it can be highlighted in the resultant fused image. According to view, sensor and Focus of input images, image fusion are categorized into five parts. 


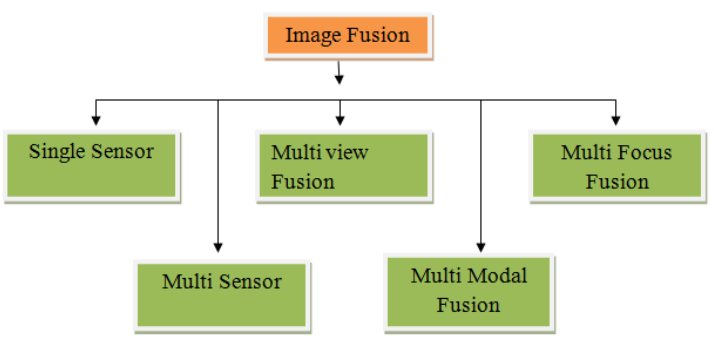

Figure 2 Types of Image Fusion

\section{APPROACHES OF FUSION TECHNIQUES}

Term fusion means to combine the information acquire in several domains. Fusion is a process which can be used to improve excellence of descriptive from a set of images. The research issues of image fusion are to improve visible quality of fused image by reducing the noise. The continuous growth in data transmission through internet and also the methods which is been developed by the hackers in recent years forced developers to developed more reliable techniques of fusion

\section{Spatial Domain Techniques \\ 2. Transform Domain Techniques}

\subsection{Spatial Domain Techniques}

In Spatial domain techniques we mainly deal with pixels of input image plane itself and all the operations done on pixel value of the input images. We know that the image is collection of pixels having $(\mathrm{x}, \mathrm{y})$ domain, Here $\mathrm{x}$ denotes the pixel position at $\mathrm{X}$ axis and $\mathrm{y}$ denotes the pixel position at $\mathrm{Y}$ axis. The Fusion techniques of spatial domain method are less complex and high payload. This method cannot even withstand at low pass filtering and common image processing attacks.

\section{Simple Maximum Method}

In this method, we take two input images. And calculate their maximum pixels corresponding to input images. The resultant fused image is generating by selecting the maximum intensity of corresponding pixels from both the input images

\section{Simple Minimum Method}

In this method, we take two input images. and calculate their minimum pixels for the resultant fused image is obtained by selecting the minimum intensity of corresponding pixels from both the input image.

\section{Simple Average Method}

In this method, we take two input images. And calculate their average pixels corresponding to input images. The resultant fused image is generating by selecting the average intensity of corresponding pixels from both the input images

\subsection{Transform Domain Techniques}

Transformed domain based fusion are more robust and reliable as compared to simple spatial domain fusion. Such algorithms are robust against simple image processing operations like low pass filtering, adjustment (brightness and contrast) blurring, etc. On the basis of transforms of images from spatial domain to frequency domain, these techniques are classified as following.

1 Image fusion using Discrete Cosine transform.

2 Image fusion using Discrete Wavelet transform.

\section{Discrete Cosine transforms}

DCT cosine transform is a transformation technique in this an image from space domain to transfer into frequency domain. In DCT method the generation of an even symmetric periodic sequence of frequency removes the discontinuity as the symmetric signal moves from one period to the next period smoothly. As a result, like the DFT, the DCT does not suffer from leakage effects image pixels. Further, symmetric extension of the signal makes its length double from the original signal, and hence, its frequency resolution is better by a factor of two. Also, due to other properties like energy compaction, the computational advantage of image fusion being real rather than complex method makes it desirable for signal processing applications like image coding, speech coding, adaptive algorithms, and for many other coding methods.

\section{Discrete wavelet transform}

The Discrete Wavelet Transform (DWT) also converts the image from the spatial domain to frequency domain. The image is divided by vertical and horizontal lines and represents the first-order of DWT, and the image can be separated with four parts those are LL1, LH1, HL1 and HH1. In additional, those four parts are represented four frequency areas in the image. For the low- frequency domain LL1 is sensitively with human eyes. In the frequency domains LH1, HL1 and HH1 have more detail Information more than frequency domain LL1.

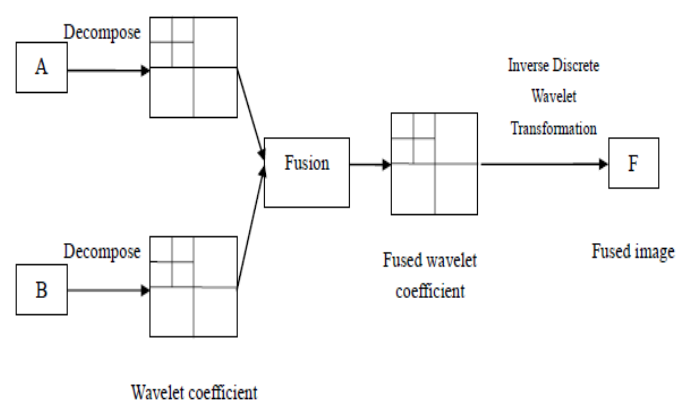

Figure 3 discrete wavelet transform (DWT) decomposition

\section{PROPOSED WORK}

In this method we take the source images. Which are in spatial domain. First this convert into transform domain by using dwt algorithm. Then apply average method on their LL band. And fuse the images. Then we again apply IDWT to get back the images in special domain. Research issues in Image Fusion are to increase efficiency in term API, Standard deviation, PSNR, robustness against visual attacks and total information.

\section{Algorithm of Proposed Hybrid DWT-DCT Fusion Technique \\ Step1:First take both input image to be fused (suppose image} name are A \& B).

Step2:Compute DWT (Discrete Wavelet Transform) of the given images which provide 4 sub bands[LL_A, LH_A, HL_A HH_A] for image A and [LL_B, LH_B, HL_B HH_B] for image $B$.

Step 3: for each sub band

Block size $=8$;

Block Bi size of $8 \times 8$ is extracted from sub band //for image A $\& \mathrm{~B}$ block is represented by $\mathrm{Bi} \_\mathrm{A}$ and $\mathrm{Bi} \_\mathrm{B}$ respectively. 
for each Block $\mathrm{i}=1$ to last Block

DCT(Bi_A) for Image A is calculated.

DCT(Bi_B) for Image B is calculated.

Average [DCT(Bi_A)+DCT(Bi_B $)] / 2$ is calculated for fusion embedding.

IDCT is calculated for fused block and place at respective fusion matrix.

end loop

end loop

Step 4: Inverse IDWT is applied for achieving image in space domain

\section{RESULTS}

The methods proposed for implementing fusion of multi focused images using DWT-DCT take the following form in general. A pair of source images namely Pepsi is taken for study and are assumed to be registered spatially. The images are transformed into wavelet domain by taking DWT-DCT transform. MATLAB codes are written to take the DWT-DCT of the two images. In each sub-band, activity measure of the two images is compared based on the above proposed fusion methods at that particular scale and space. A fused wavelet transform is created by taking pixels from that wavelet transform that shows greater activity at the pixel locations. The inverse DWT-DCT is the fused image with clear focus on the whole image. Image Fusion in Wavelet transform domain has higher robustness against statistical attacks compared to image fusion in spatial domain and Discrete Cosine Transform domain. While Spatial and Discrete cosine transform technique have a PSNR better than other technique.

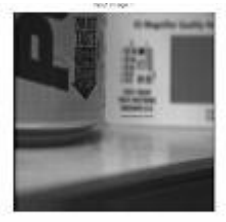

(A)

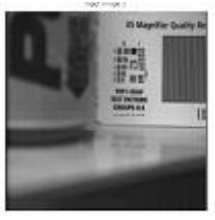

(B)

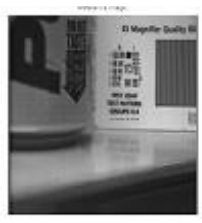

(C)
Figure 4. A, B- Input Images, C- Fused image using DWT+DCT

\subsection{Results of colour images}

Table 1: Result of multi focus image with $256 * 256$ pixels using proposed Hybrid DWT-DCT

\begin{tabular}{|l|l|l|l|l|l|l|}
\hline $\begin{array}{l}\text { Measu } \\
\text { res }\end{array}$ & API & SD & $\begin{array}{l}\text { PSN } \\
\text { R }\end{array}$ & AG & $\begin{array}{l}\text { Entro } \\
\text { py }\end{array}$ & MIF \\
\hline $\begin{array}{l}\text { Cold } \\
\text { drink. } \\
\text { jpg }\end{array}$ & $\begin{array}{l}105.25 \\
54\end{array}$ & $\begin{array}{l}60.06 \\
64\end{array}$ & $\begin{array}{l}35.14 \\
97\end{array}$ & $\begin{array}{l}12.11 \\
55\end{array}$ & $\begin{array}{l}15.16 \\
78\end{array}$ & $\begin{array}{l}39.96 \\
06\end{array}$ \\
\hline $\begin{array}{l}\text { Leena. } \\
\text { jpg }\end{array}$ & $\begin{array}{l}151.95 \\
59\end{array}$ & $\begin{array}{l}60.90 \\
99\end{array}$ & $\begin{array}{l}35.08 \\
91\end{array}$ & $\begin{array}{l}10.67 \\
76\end{array}$ & $\begin{array}{l}13.62 \\
52\end{array}$ & $\begin{array}{l}\text { Infini } \\
\text { ty }\end{array}$ \\
\hline
\end{tabular}

\subsection{Results of gray scale images}

Table 2 Result of multi focus image with $256 * 256$ pixels using proposed Hybrid DWT-DCT

\begin{tabular}{|l|l|l|l|l|}
\hline Measures & API & SD & PSNR & $\begin{array}{l}\text { Entrop } \\
\mathrm{y}\end{array}$ \\
\hline Pepsi.jpg & $\begin{array}{l}97.806 \\
5\end{array}$ & 42.6922 & $\begin{array}{l}36.632 \\
5\end{array}$ & $\begin{array}{l}13.946 \\
1\end{array}$ \\
& & & & \\
\hline
\end{tabular}

\begin{tabular}{|l|l|l|l|l|}
\hline MRI.jpg & $\begin{array}{l}34.622 \\
7\end{array}$ & 44.0227 & $\begin{array}{l}36.499 \\
2\end{array}$ & $\begin{array}{l}12.165 \\
2\end{array}$ \\
\hline Leena.jpg & $\begin{array}{l}99.134 \\
7\end{array}$ & 46.7739 & $\begin{array}{l}36.236 \\
0\end{array}$ & $\begin{array}{l}14.807 \\
6\end{array}$ \\
& & & & \\
\hline Saras.jpg & $\begin{array}{l}227.67 \\
79\end{array}$ & 45.9000 & $\begin{array}{l}37.567 \\
3\end{array}$ & 8.0539 \\
& & & & \\
\hline
\end{tabular}

Here we measure six parameters for image quality metrics. In this report we use hybrid DWT-DCT technique for image fusion. By using this technique we measure six parameters for coloured image as well as gray scale images. After calculating this results we also analysis this parameters to different existing techniques. These are followings.

\subsection{Comparison of Proposed Results with Existing Results}

Table 3 Comparative Result of Proposed Technique with Existing technique for Leena.jpg coloured image

\begin{tabular}{|l|l|l|l|l|}
\hline $\begin{array}{l}\text { S. } \\
\text { No. }\end{array}$ & Method & $\begin{array}{l}\text { Existing } \\
\text { PSNR } \\
\text { [9] }\end{array}$ & Method & $\begin{array}{l}\text { Proposed } \\
\text { PSNR }\end{array}$ \\
\hline 1 & DCT & 26.3124 & DWT+DCT & 35.0891 \\
\hline 2 & WT & 21.8177 & & \\
\hline 3 & IWT & 23.8177 & & \\
\hline
\end{tabular}

Here we compare coloured Leena.jpg image with existing technique DCT, WT, IWT given by Mr. Vipin Wani, Prof. Mukesh Baghe, Prof. Hitesh Gupta [9] calculate PSNR $26.3124,21.8177,23.8177$ respectively. This result is comparatively low to proposed method of hybrid DWT+DCT method. Therefore we conclude that Hybrid DWT-DCT give better performance of coloured fused image with existing methods.

Table 4 Comparative Result of Proposed Technique with Existing technique

\begin{tabular}{|l|l|l|l|l|l|}
\hline $\begin{array}{l}\text { S. } \\
\text { N } \\
\text { o. }\end{array}$ & Image & $\begin{array}{l}\text { Metho } \\
\text { d }\end{array}$ & $\begin{array}{l}\text { Existi } \\
\text { ng } \\
\text { PSNR }\end{array}$ & $\begin{array}{l}\text { Meth } \\
\text { od }\end{array}$ & $\begin{array}{l}\text { Propos } \\
\text { ed } \\
\text { PSNR }\end{array}$ \\
\hline 1 & $\begin{array}{l}\text { Pepsi. } \\
\text { jpg }\end{array}$ & $\begin{array}{l}\text { HDW } \\
\text { T }\end{array}$ & $\begin{array}{l}36.41 \\
66\end{array}$ & $\begin{array}{l}\text { DWT } \\
\text {-DCT }\end{array}$ & $\begin{array}{l}\mathbf{3 6 . 6 3 2} \\
\mathbf{5}\end{array}$ \\
\hline 2 & MRI.j & PCA & 25.48 & DWT & $\mathbf{3 6 . 4 9 1}$ \\
& pg & AVG & {$[5]$} & -DCT & $\mathbf{2}$ \\
& & DWT & 17.07 & & \\
& & & 11 & & \\
& & & $26]$ & & \\
\hline 3 & Leena & DCT & 26.31 & DWT & $\mathbf{3 6 . 2 3 6}$ \\
& .jpg & & 24 & -DCT & $\mathbf{0}$ \\
& & & {$[9]$} & & \\
& & & 28.30 & & \\
& & & & & \\
\hline
\end{tabular}

Here we compare different multi focus images with existing technique to proposed technique. First we compare Existing Pepsi image using HDWT method calculate PSNR 36.4166 to proposed DCT-DWT method Calculate PSNR 36.6325. 
Second we compare Existing MRI image using PCA, Average DWT method calculate PSNR 25.48, 17.0711 to proposed DCT-DWT method Calculate PSNR 36.4912. third we compare Existing Leena image using DCT method calculate PSNR 26.3124 [9] , 28.30 [10] to proposed DCTDWT method Calculate PSNR 36.2360. This is higher to existing technique. Then we conclude that Hybrid DCT-DWT gives better result as compared to DCT, PCA and HDWT Technique.

\subsection{Analysis Graph of Results}

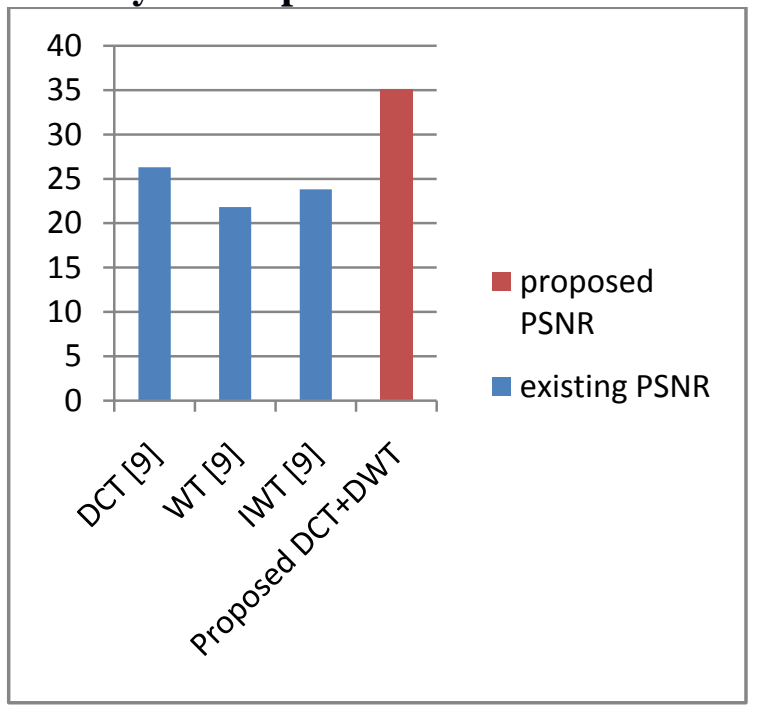

Figure 5 Analysis Graph of coloured Leena image with Existing Technique [9] And Proposed Technique DWTDCT

This analysis does for PSNR value of coloured leena image in between Existing Method by Mr. Vipin Wani, Prof. Mukesh Baghe, Prof. Hitesh Gupta [9] and Proposed Hybrid DWTDCT method. And we conclude that Proposed Method gives Better result as compare to Existing Technique

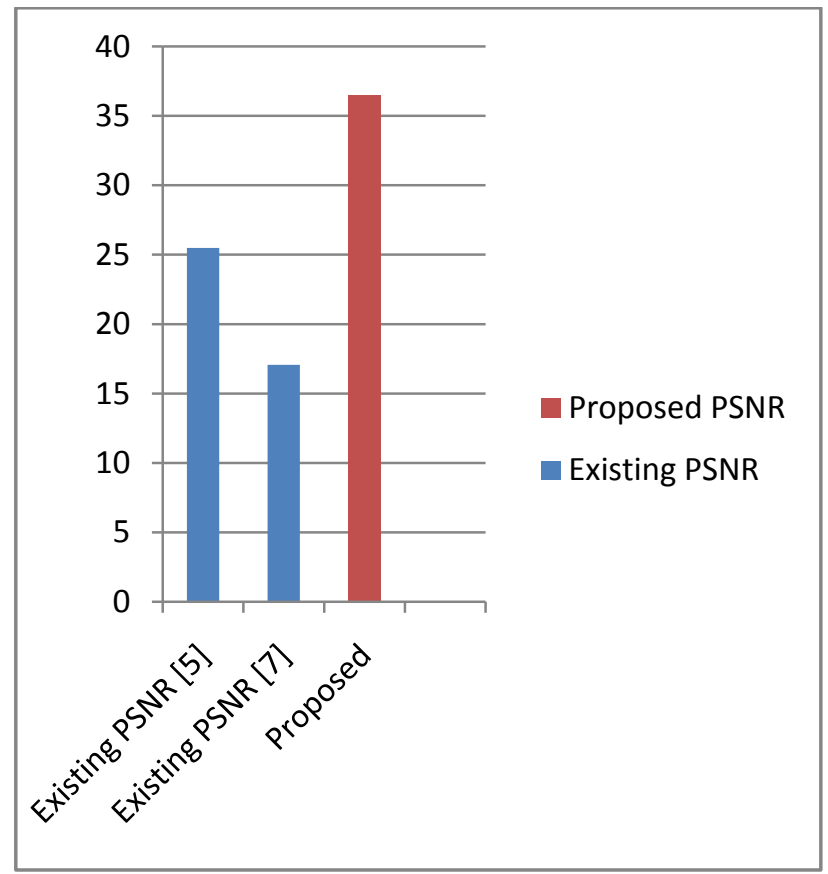

Figure 6 Analysis Graph of image MRI.jpg with Existing Technique [5] [7] And Proposed Technique
This analysis does for PSNR value of MRI image in between Existing Method Srinivasa Rao Dammavalam Seetha Maddala and Krishna Prasad MHM [7], Kusum Rani, Reecha Sharma [5] and Proposed Hybrid DWT-DCT method. And we conclude that Proposed Method gives Better result as compare to Existing Technique.

\section{CONCLUSION}

The Proposed algorithm presents Hybrid DWT-DCT Digital image fusion algorithm. Proposed method exploits strength of two combined transform domain techniques DCT \& DWT to obtain better image quality of fused image.

From the results achieved by different technique mentioned in above it is observed that proposed image fusion techniques provide higher PSNR compared to individual DWT, DCT based existing image fusion technique so proposed work is better for image quality than existing image fusion technique. Proposed technique has lower standard deviation and higher PSNR compared to existing DWT and DCT technique so proposed technique provide more quality image compared to existing technique.

\section{FUTURE WORK}

Proposed technique can be modified for further enhancement of image quality parameter of fused image and performances are calculated against different attacks. Till now, we have worked upon grey scale and coloured image but we can perform fusion on animated image and video as well in future.

\section{REFERENCES}

[1.] James, Alex Pappachen, and Belur V. Dasarathy. "Medical image fusion: A survey of the state of the art." Information Fusion 19 (2014): 4-19.

[2.] Abuturab, Muhammad Rafiq. "Multiple color-image fusion and watermarking based on optical interference and wavelet transform." Optics and Lasers in Engineering (2016).

[3.] Galande, Ashwini, and Ratna Patil. "The art of medical image fusion: A survey." Advances in Computing, Communications and Informatics (ICACCI), 2013 International Conference on. IEEE, 2013.

[4.] Li, Shutao, Bin Yang, and Jianwen Hu. "Performance comparison of different multi-resolution transforms for image fusion." Information Fusion 12, no. 2 (2011): 7484

[5.] Kusum Rani, Reecha Sharma , "Study of different image fusion algorithm" International Journal of Emerging Technology And Advanced Engineering ( IJETAE) volume 3, issue 5, may 2013

[6.] K. Kannan , S. Arumuga Perumal , " Fusion of multifocused images using HDWT for machine vision " International Journal Of Computer Science And Engineering. Volume 2, no 5, ( 2011 ) ISSN 0976 - 5166

[7.] Srinivasa Rao Dammavalam, Seetha Maddala and Krishna Prasad MHM, "Quality assessment of pixellevel image Fusion using fuzzy logic" International Journal on Soft Computing ( IJSC ) Vol.3, No.1, February 2012

[8.] S. S. Bedi, Rati Khandelwal "Comprehensive and Comparative Study of Image Fusion Techniques" International Journal of Soft Computing and Engineering (IJSCE) ISSN: 2231-2307, Volume-3, Issue-1, March 
2013

[9.] Mr. Vipin Wani, Prof. Mukesh Baghe, Prof. Hitesh Gupta, "A Comparative Study of Image Fusion Technique Based on Feature Using Transforms Function" International Journal of Emerging Technology and Advanced Engineering. ISSN 2250-2459, ISO 9001:2008, Volume 3, Issue 11, November 2013

[10.]Ms. Rubeena Vohra Dr. K.C. Tiwari, "A Review and Assessment of Fusion Algorithms on Gray Scale Images" International Journal of Electronics, Electrical and Computational System IJEECS ISSN 2348-117X Volume 4, Special Issue September 2015

[11.]PreetKaur, Geetulalit, "Comparative Analysis of DCT, DWT \& LWT for Image Compression" International Journal of Innovative Technology and Exploring Engineering (IJITEE) Volume-1, Issue-3, August 2012
[12.] Shalima , Dr. Rajinder Virk, "Review Of Image Fusion Techniques" International Research Journal of Engineering and Technology (IRJET) ISSN: 2395 -0056, Volume: 02 Issue: 03 | June-2015

[13.]Harmandeep Kaur, Er. Jyoti Rani, “Analytical Comparison of Various Image Fusion Techniques"International Journal of Advanced Research in Computer Science and Software Engineering, ISSN: 2277 128X, Volume 5, Issue 5, May 2015

[14.]Dr.S.S.Bedi, Mrs.Jyoti Agarwal, Pankaj Agarwal, "Image Fusion Techniques and Quality Assessment Parameters for Clinical Diagnosis: A Review" , International Journal of Advanced Research in Computer and Communication Engineering , ISSN (Online) : 2278-1021, Vol. 2, Issue 2, February 2013 